\section{(6) OPEN ACCESS}

\title{
The acute management of haemorrhage, surgery and overdose in patients receiving dabigatran
}

\author{
Raza Alikhan, ${ }^{1}$ Rachel Rayment, ${ }^{1}$ David Keeling, ${ }^{2}$ Trevor Baglin, ${ }^{3}$ Gary Benson, ${ }^{4}$ \\ Laura Green ${ }^{5}$ Scott Marshall, ${ }^{6}$ Raj Patel, ${ }^{7}$ Sue Pavord, ${ }^{8}$ Peter Rose, ${ }^{9}$ Campbell Tait ${ }^{10}$
}

For numbered affiliations see end of article.

\section{Correspondence to} Dr Raza Alikhan, Haemophilia and Thrombosis Centre, University Hospital of Wales, Heath Park, Cardiff CF14 4XW, UK; raza.alikhan@wales.nhs.uk

Received 18 September 2012 Revised 6 January 2013 Accepted 7 January 2013 Published Online First 22 February 2013

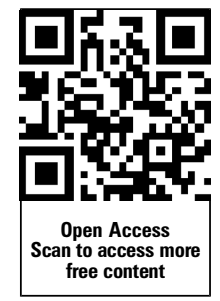

To cite: Alikhan $\mathrm{R}$, Rayment R, Keeling $D$, et al. Emerg Med J 2014;31: 163-168.

\section{ABSTRACT}

Dabigatran is an oral direct thrombin inhibitor (DTI) licensed for stroke prevention in atrial fibrillation and likely to be soon approved in Europe for treatment of venous thrombosis. Predictable pharmacokinetics and a reduced risk of intracranial haemorrhage do not negate the potential risk of haemorrhage. Unlike warfarin, there is no reversal agent and measurement of the anticoagulant effect is not 'routine'. The prothrombin time/international normalised ratio response to dabigatran is inconsistent and should not be measured when assessing a patient who is bleeding or needs emergency surgery. The activated partial thromboplastin time (APTT) provides a qualitative measurement of the anticoagulant effect of dabigatran. Knowledge of the time of last dose is important for interpretation of the APTT. Commercially available DTI assays provide a quantitative measurement of active dabigatran concentration in the plasma. If a patient receiving dabigatran presents with bleeding: omit/delay next dose of dabigatran; measure APTT and thrombin time (consider DTI assay if available); administer activated charcoal, with sorbitol, if within $2 \mathrm{~h}$ of dabigatran ingestion; give tranexamic acid (1 $\mathrm{g}$ intravenously if significant bleeding); maintain renal perfusion and urine output to aid dabigatran excretion. Dabigatran exhibits low protein binding and may be removed by dialysis. Supportive care should form the mainstay of treatment. If bleeding is life/limb threatening, consider an additional haemostatic agent. There is currently no evidence to support the choice of one haemostatic agent (FEIBA, recombinant factor VIla, prothrombin complex concentrates) over another. Choice will depend on access to and experience with available haemostatic agent(s).

Dabigatran etexilate is an oral direct thrombin inhibitor (DTI), which is rapidly absorbed after oral administration, reaching a peak plasma concentration and maximal anticoagulant effect within 2-3 h. ${ }^{1}$ Dabigatran displays linear pharmacokinetics, over a wide range of doses, which allows it to be given in a fixed-dose regimen without the need for routine coagulation monitoring. ${ }^{2} 3$ Although the risk of spontaneous intracranial haemorrhage $(\mathrm{ICH})$ is reduced, ${ }^{4}$ patients receiving this drug are fully anticoagulated and are at risk of bleeding, particularly in association with trauma ${ }^{5}$ and surgery and after the development of renal failure. ${ }^{6}$

The RE-LY Study ${ }^{2}$ compared two doses of dabigatran $(150 \mathrm{mg}$ and $110 \mathrm{mg}$ twice a day) with dose-adjusted warfarin for stroke prevention in atrial fibrillation. The primary efficacy outcome of the study was stroke or systemic embolisation. The $150 \mathrm{mg}$ dose of dabigatran was superior to warfarin
(1.11\% vs $1.71 \%$, relative risk (RR) 0.65 (95\% CI 0.52 to 0.81$) \mathrm{p}<0.001)$. The primary safety outcome was major bleeding events. The rate of major bleeding was significantly less in the dabigatran $110 \mathrm{mg}$ group compared with warfarin ( $2.87 \%$ vs $3.57 \%, p=0.003$, respectively), but was similar to warfarin in the dabigatran $150 \mathrm{mg}$ group $(3.32 \%$ vs $3.57 \%)$ per year with a RR of 0.93 (95\% CI 0.81 to 1.07 ; $\mathrm{p}=0.32$ ).

In England and Wales, The National Institute for Health and Clinical Excellence, ${ }^{7}$ and in Scotland the Scottish Medicines Consortium, ${ }^{8}$ have recently recommended the anticoagulant, dabigatran, to be considered as an option for the prevention of stroke and systemic embolism in people with atrial fibrillation. As prescriptions for dabigatran increase, clinicians will need to be prepared to deal with complications such as bleeding (spontaneous and trauma-related) as well as patients who require semi-urgent or urgent surgery.

The recently published 9th American College of Chest Physicians clinical practice guidelines ${ }^{9}$ state that 'there is insufficient clinical experience to firmly guide the management of major bleeding, suspected overdose, urgently needed surgery, or urgent invasive diagnostic or therapeutic procedures in patients who are taking this new drug'. These comments offer little solace or guidance to a clinician faced with having to manage one of the above described scenarios. This paper aims to provide practical advice to clinicians on the laboratory investigation and acute management of patients presenting while anticoagulated with dabigatran.

\section{COAGULATION ASSAYS: MEASURING THE EFFECT OF DABIGATRAN \\ Prothrombin time /international normalised ratio}

Vitamin $\mathrm{K}$ antagonists, such as warfarin, reduce the levels of the coagulation factors II, VII, IX and X. ${ }^{9}$ The prothrombin time (PT) is particularly sensitive to a reduction in factor VII, and, as a result, treatment with vitamin $\mathrm{K}$ antagonists results in prolongation of the PT. This is usually expressed as a standardised ratio (the international normalised ratio (INR)), which is used to monitor the therapeutic anticoagulant effect of warfarin. ${ }^{10}$

The PT/INR is insensitive to dabigatran at therapeutic levels. ${ }^{11}$ It may be prolonged by supratherapeutic levels of dabigatran, but the results will vary widely between laboratories, because of variation in the sensitivity of different reagents to the effect of dabigatran. ${ }^{11}$ Neither a laboratory-based nor 
point-of-care PT/INR can be used to assess the anticoagulant effect of dabigatran.

\section{Activated partial thromboplastin time}

The activated partial thromboplastin time (APTT) provides a measure of the intrinsic (factors VIII, IX and XI) and common (factors II, V, X and fibrinogen) coagulation pathway. The APTT can be used to monitor the anticoagulant effect of unfractionated heparin. ${ }^{12}$ The APTT displays a curvilinear dose response to increasing plasma concentrations of dabigatran ${ }^{11}$ and may be used to demonstrate that a patient is anticoagulated with dabigatran-for example, if a patient is bleeding or needs surgery. However, it does not provide a quantitative measurement and should not be used for routine monitoring.

In the stroke prevention trial, ${ }^{2}$ the APTT ratio was $1.4-2.3$ in patients receiving $150 \mathrm{mg}$ twice a day, and 1.3-2.1 in those receiving $110 \mathrm{mg}$ twice a day, with a peak effect seen $2 \mathrm{~h}$ after administration. ${ }^{13}$ Prolongation of the APTT is maintained for at least $8-12 \mathrm{~h}$, with a measurable effect still seen in some patients after 24 h. $^{1}$ If the APTT is prolonged by $2-3$-fold at trough (when the next dose is due), there is a higher risk of bleeding. ${ }^{14} 15$ Therefore, knowledge of the time of last dose is important for interpretation of the APTT, particularly if an invasive procedure is planned.

\section{Thrombin time}

The thrombin time (TT) provides a measurement of the conversion of fibrinogen into fibrin (clot). The TT displays a linear time response to increasing plasma concentrations of dabigatran. ${ }^{1}$ In general, the TT does not form part of a 'routine' coagulation profile and may not be readily available in clinical practice. In addition, the TT is exquisitely sensitive to the presence of dabigatran, and samples may be unclottable at therapeutic levels, making it unsuitable as a quantitative assay. ${ }^{14}{ }^{15}$ Conversely, a normal TT indicates an absence of dabigatran anticoagulant effect and could therefore be used to exclude the drug as a cause of haemorrhage.

\section{DTI assay}

The commercially available HEMOCLOT (HYPHEN BioMed, France $)^{16}$ DTI assay avoids the over-sensitivity displayed by standard TT assays. The clotting time is directly related to the concentration of active dabigatran in the patient's plasma and can be used to measure the anticoagulant activity of dabigatran. A DTI assay may be of use in assessing patients presenting with bleeding or in those that require an urgent invasive procedure or surgery.

\section{MANAGEMENT OF BLEEDING}

There are currently no antidotes available for reversing the anticoagulant effect of dabigatran, although preclinical work is underway to develop a neutralising, dabigatran-specific monoclonal antibody. ${ }^{17}$ If a patient presents with bleeding, dabigatran therapy should be discontinued until appropriate investigations are completed and the patient is stabilised (figure 1). ${ }^{18-20}$

\section{Activated charcoal}

Activated charcoal is a processed form of carbon with a large porous surface area available to bind to oral drugs such as dabigatran $^{21} 22$ and reduce absorption from the gastrointestinal tract. Binding to charcoal is reversible; sorbitol is often added for its laxative effect to speed transit through the intestines. Since dabigatran is rapidly absorbed after ingestion, activated charcoal is likely to be of greatest benefit within $2 \mathrm{~h}$ of ingestion. Clinicians should bear in mind that absorption of concomitant oral medication will also be impaired by administration of charcoal.

Figure 1 Management of bleeding patient anticoagulated with dabigatran. ${ }^{18-20}$ APTT, activated partial thromboplastin time; TT, thrombin time; eGFR, estimated glomerular filtration rate; $\mathrm{CrCl}$, creatinine clearance; $\mathrm{BP}$, blood pressure; i.v. intravenous; Hb, haemoglobin; Plt, platelet; CNS, central nervous system; FEIBA, factor eight inhibitor bypassing activity; PCC, prothrombin complex concentrate; rFVIla, recombinant factor VIla. 


\section{Tranexamic acid}

Tranexamic acid inhibits fibrinolysis by inhibiting the binding of plasmin to fibrin. Tranexamic acid has been shown to reduce bleeding after tissue injury associated with surgery ${ }^{23}$ and after trauma. ${ }^{24}$ Based on the results of more than 200 randomised control trials, the efficacy of anti-fibrinolytic drugs does not appear to be offset by serious side effects. ${ }^{23}$ In the event of major bleeding with dabigatran, $1 \mathrm{~g}$ tranexamic acid should be given intravenously.

\section{General supportive measures}

Maintaining renal perfusion and urine output can aid elimination of dabigatran, $80 \%$ of which is excreted through the kidneys unchanged. ${ }^{25}$ Mechanical methods to arrest bleeding, including compression, tamponade, surgery and radiological intervention, are essential components of managing bleeding events.

Since the half-life of dabigatran is short $(12-17 \mathrm{~h}),{ }^{1}{ }^{15}$ it is envisaged that the majority of bleeding episodes will be managed by supportive methods alone. However, if bleeding continues or is life threatening, then an additional haemostatic agent should be considered. It must be remembered that there is no specific antidote for dabigatran and there is no ideal haemostatic agent for this indication.

\section{Dialysis}

Dabigatran exhibits low protein binding and therefore it may be possible to remove it from the circulation by dialysis. The plasma concentration of dabigatran can be reduced by $50-60 \%$ after $4 \mathrm{~h}$ of dialysis. ${ }^{26} 27$ Unfortunately, emergency access to haemodialysis may be limited, and the technical issues surrounding establishing venous access in an anticoagulated patient means that this treatment option may only be available to a few patients.

\section{Haemostatic agents that may be of use in the management of bleeding}

Prothrombin complex concentrates

Prothrombin complex concentrates (PCCs) contain factors II (prothrombin), VII, IX and X, which are the vitamin K-dependent factors affected by warfarin therapy. Dabigatran directly inhibits thrombin, and, although administration of a PCC will not influence this effect, it may provide more substrate (prothrombin) to increase thrombin generation, which is essential for the formation of a fibrin blood clot.

A number of animal studies have found that the PCCs, Beriplex $^{28-30}$ and Octaplex, ${ }^{29}$ result in haemostasis and reversal of blood loss after supratherapeutic doses of dabigatran. In a murine ICH model, a PCC resulted in cessation of bleeding associated with dabigatran. It also prevented haematoma expansion and significantly reduced mortality. ${ }^{30}$ In contrast, in a study of human healthy volunteers, a PCC (Cofact) failed to normalise the prolonged APTT or TT caused by dabigatran, ${ }^{31}$ although the effect on bleeding was not assessed in this study. It is important to note that, in the animal models where a clinical effect was seen, there was no improvement in the laboratory tests. ${ }^{29} 3032$ The inability to measure the effect of PCC administration by laboratory tests may result in larger doses of PCC being given than necessary, leading to the potential for thrombotic complications.

\section{FEIBA}

FEIBA (which has Factor Eight Inhibitor Bypassing Activity) is currently used as a haemostatic agent for patients with antibodies to factor VIII. Activated factor $\mathrm{X}$ and prothrombin appear to be the main components in FEIBA responsible for its haemostatic action, ${ }^{33}$ and they enable the generation of thrombin, independently of factor VIII. As in the case of PCCs, this thrombin may still be inhibited by dabigatran.

In a rat tail bleeding model, FEIBA significantly reduced the bleeding time associated with dabigatran. ${ }^{22}$ As with the PCC studies, the APTT remained prolonged after FEIBA administration despite the reduction in bleeding time. There is a single case report of the successful use of FEIBA in the management of a life-threatening bleeding event associated with dabigatran. ${ }^{34}$ It is likely that the prothrombotic effect of FEIBA is greater than that of PCCs because of the coagulation factors being activated and that, if used, much smaller doses will be required than those used for treating patients with haemophilia.

\section{Recombinant factor VIla}

Recombinant factor VIIa (rFVIIa) is another bypassing haemostatic agent used for the management of haemophiliac patients with inhibitors. It is able to directly activate factors IX and X resulting in a burst of thrombin generation (and thus fibrin clot formation) independently of factor VIII. ${ }^{35}$ As with the other haemostatic agents, the generation of thrombin may in part overcome the effect of dabigatran.

rFVIIa has also been shown to reduce dabigatran-induced bleeding in a rat tail bleeding model with partial normalisation of the APTT. ${ }^{29}$ However, in contrast with PCCs, rFVIIa failed to reduce haematoma expansion or have an impact on mortality in a murine ICH model. ${ }^{30}$

Following anecdotal evidence, the off-label use of rFVIIa for non-haemophiliac patients who are bleeding has gained some favour in the past decade. However, there is concern that rFVIIa is associated with an increased risk of thrombotic complications. A review of 35 randomised control trials of the off-label use of rFVIIa identified a significant increase in the risk of arterial events, particularly in the elderly. ${ }^{36}$

\section{Surgery/invasive procedure}

When a patient receiving dabigatran requires an elective invasive procedure, the consequences of bleeding if anticoagulation is continued, with the risk of thrombosis if it is omitted, need to be considered. In addition, as dabigatran is predominantly eliminated via the renal pathway, this will need to be taken into account when deciding for how long to stop dabigatran before a procedure (table 1 ).

For patients who require urgent surgery, discussion with the patient's surgeon and anaesthetist are of paramount importance (figure 2). A baseline assessment of coagulation should be

Table 1 Discontinuation rules for dabigatran before invasive or surgical procedure 81415

\begin{tabular}{llll}
\hline & & \multicolumn{2}{l}{ Stop dabigatran before elective surgery } \\
\cline { 3 - 4 } $\begin{array}{l}\text { Renal function } \\
(\mathrm{CrCl}(\mathrm{ml} / \mathrm{min}))\end{array}$ & $\begin{array}{l}\text { Estimated } \\
\text { half-life(h) }\end{array}$ & $\begin{array}{l}\text { Major surgery or } \\
\text { high bleeding risk* }\end{array}$ & $\begin{array}{l}\text { Non-major surgery } \\
\text { or standard riskt }\end{array}$ \\
\hline$>80$ & $\sim 13$ & $48 \mathrm{~h}$ before & $24 \mathrm{~h}$ before \\
$>50$ and $<80$ & $\sim 15$ & $48-72 \mathrm{~h}$ before & $24-48 \mathrm{~h}$ before \\
$>30$ and $<50$ & $\sim 18$ & $96 \mathrm{~h}$ before & $48-72 \mathrm{~h}$ before \\
\hline
\end{tabular}

*Examples of major surgery/high bleeding risk: cardiothoracic surgery, neurosurgery, major abdominal or pelvic surgery, major orthopaedic surgery, insertion of cardiac pacemaker/defibrillator.

†Examples of non-major surgery/standard risk: uncomplicated laparoscopic procedure, cardiac catheterisation/ablation therapy.

$\mathrm{CrCl}$, creatinine clearance. 


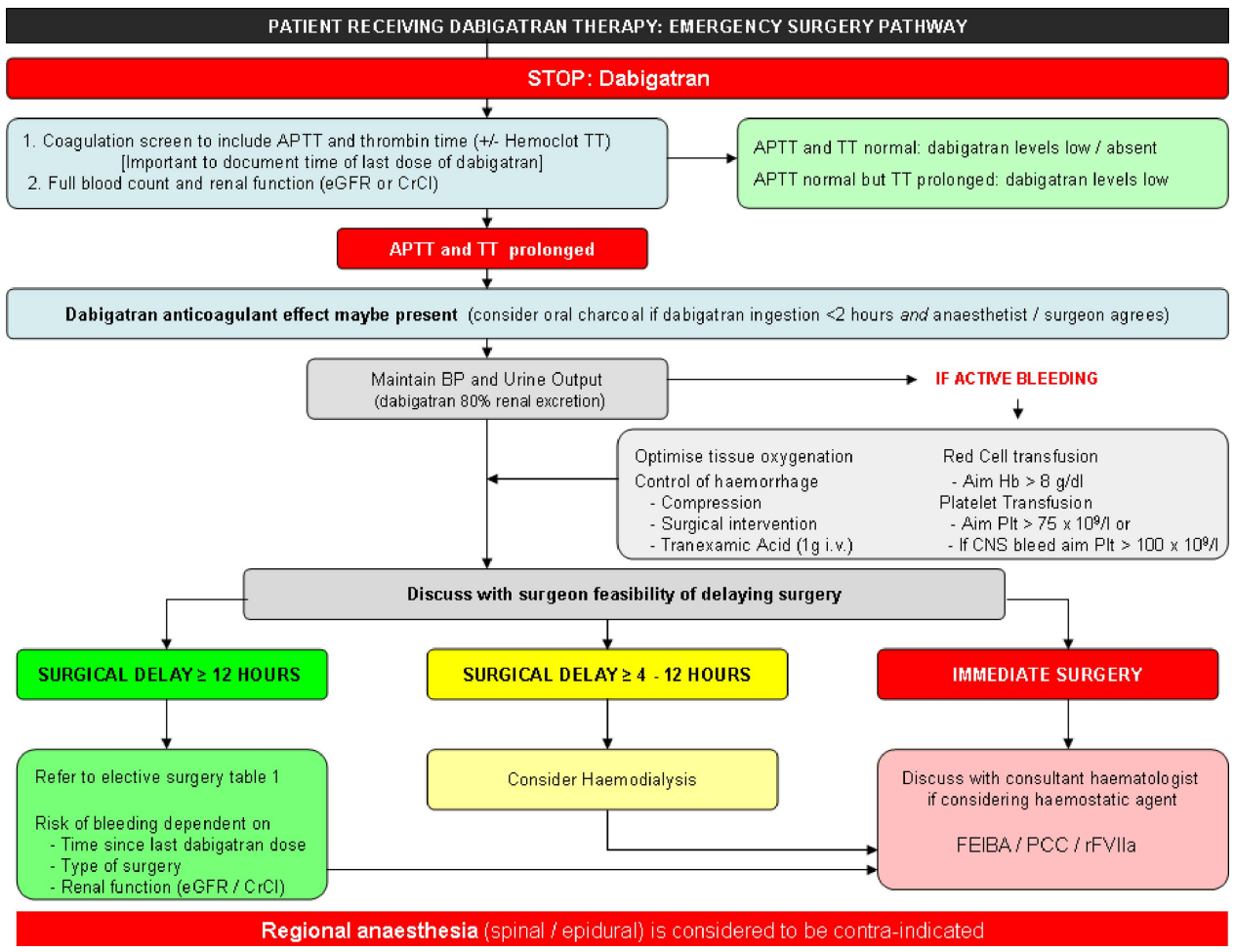

Figure 2 Management of patient anticoagulated with dabigatran requiring urgent surgery. APTT, activated partial thromboplastin time; TT, thrombin time; eGFR, estimated glomerular filtration rate; $\mathrm{CrCl}$, creatinine clearance; BP, blood pressure; i.v. intravenous; $\mathrm{Hb}$, haemoglobin; Plt, platelet; CNS, central nervous system; FEIBA, factor eight inhibitor bypassing activity; PCC, prothrombin complex concentrate; rFVIla, recombinant factor VIla.

performed, including the APTT. A normal APTT suggests that the bleeding risk is low. A prolonged APTT may reflect the presence of dabigatran ${ }^{11}$ or, particularly in the setting of trauma or sepsis, a coagulopathy. A DTI assay, if available, could provide additional information about the risk of bleeding. ${ }^{14}$

Prolongation of the APTT and/or a measurable therapeutic dabigatran concentration in the patient's plasma should prompt a discussion as to the feasibility of delaying surgery. A delay of $24 \mathrm{~h}$ in a patient with normal renal function will allow the concentration of dabigatran in the plasma to fall by $75 \%$. If urgent surgery is required, particularly within a few hours of dabigatran ingestion, an increased risk of bleeding is likely.

To support drug elimination from the kidneys, renal perfusion and urine output must be maintained. If a patient has taken

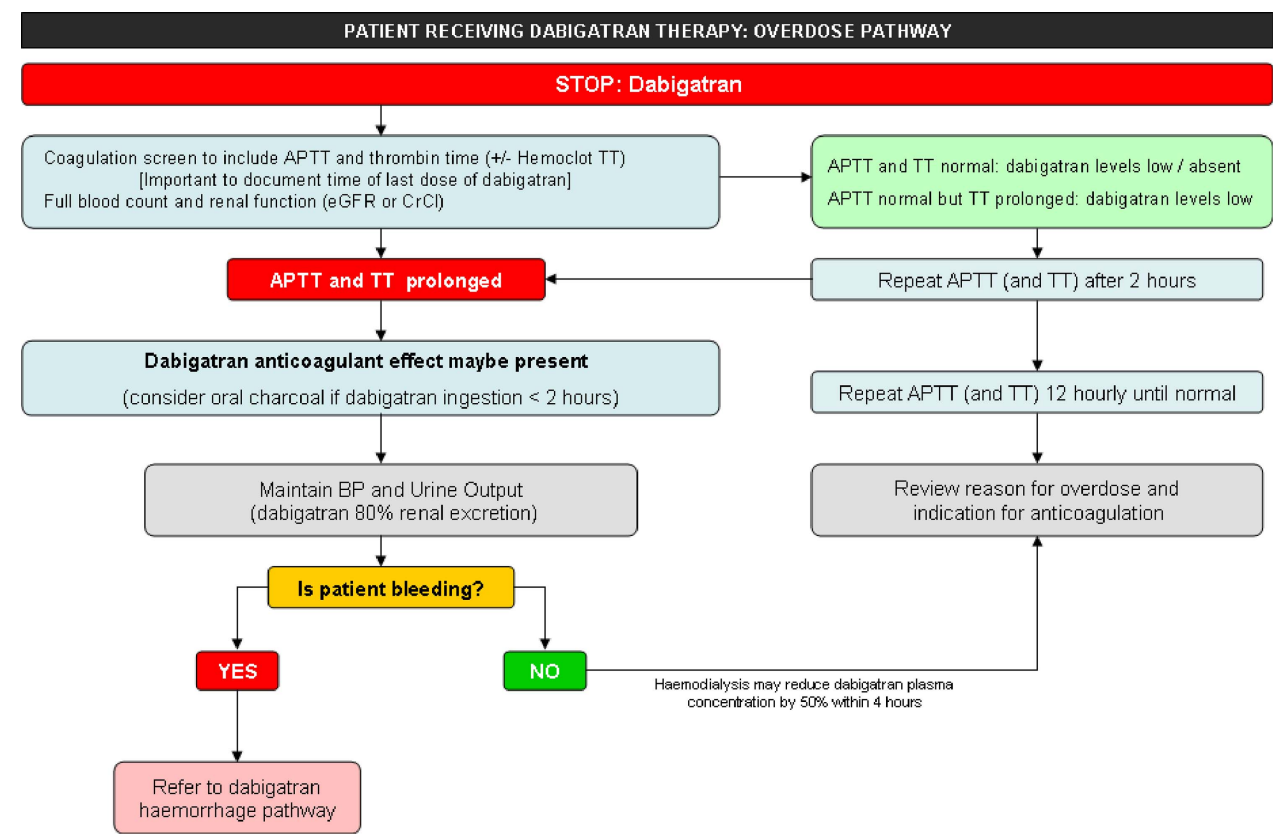

Figure 3 Management of patient after an overdose of dabigatran. APTT, activated partial thromboplastin time; TT, thrombin time; eGFR, estimated glomerular filtration rate; $\mathrm{CrCl}$, creatinine clearance; BP, blood pressure; i.v. intravenous; Hb, haemoglobin; Plt, platelet; CNS, central nervous system. 
dabigatran in the preceding $2 \mathrm{~h}$ and there is no surgical or anaesthetic contraindication, activated charcoal may be considered as an option to reduce dabigatran absorption.

Regional anaesthesia should be avoided in a patient who requires surgery within $24-48 \mathrm{~h}$ of ingestion of a therapeutic dose of dabigatran (even if the APTT/DTI are normal). ${ }^{37}$ A decision to perform regional anaesthesia, on the basis of the risk of general anaesthesia, should be thoroughly discussed with the patient/relatives.

If bleeding occurs, then supportive measures should be used, with crystalloids, red blood cells, platelets and plasma (figure 2). Additional haemostatic agents may be considered during or after surgery if bleeding continues. There is currently no evidence to support the preoperative administration of a haemostatic agent.

After surgery, the risk of bleeding associated with the procedure should be measured against the risk of thrombosis associated with withholding anticoagulation. A therapeutic dose of dabigatran should be withheld for 24-72 h after surgery, according to the patient's individual risk of bleeding and thrombotic risk. Routine thromboprophylaxis with a licensed anticoagulant may be considered $6-8 \mathrm{~h}$ after the operation and continued until dabigatran therapy is re-introduced.

\section{Overdose}

In the event of accidental or intentional overdose, a baseline APTT and TT or DTI assay should be performed (figure 3). If normal, they should be repeated after $2 \mathrm{~h}$, since plasma levels of dabigatran peak within $2 \mathrm{~h}$ of ingestion. If the time of ingestion is known, a $12 \mathrm{~h}$ APTT trough measurement can be used as an indication of the patient's risk of bleeding; a trough APTT $>2-3$ times the upper limit of normal or a plasma concentration of $>200 \mathrm{ng} /$ $\mathrm{ml}$ is likely to be associated with a higher risk of bleeding. ${ }^{14} 15$

Activated charcoal with sorbitol should be considered for all patients with suspected dabigatran overdose. It is likely to be of most benefit administered within $2 \mathrm{~h}$ of ingestion. The patient should be warned of the potential for black stools, and the medical team should also be made aware, to avoid misdiagnosis of melaena.

After an overdose, it may be possible to remove dabigatran from the circulation by haemodialysis, which reduces the plasma concentration of dabigatran by $50-60 \%$ within $4 \mathrm{~h}$ of dialysis. $^{26} 27$

\section{CONCLUSION}

Despite the undoubted advantages offered by the new oral anticoagulants such as dabigatran, it would be naive of us to think that we will not be faced at some point in the near future with a patient anticoagulated with this new drug who requires urgent surgery or treatment for bleeding. At present, there is little published evidence to guide clinicians. Our review and practical suggestions are aimed at filling that void until such time that studies addressing these issues are published or an antidote to dabigatran is marketed.

\footnotetext{
Author affiliations

${ }^{1}$ Haemophilia and Thrombosis Centre, University Hospital of Wales, Cardiff, UK

${ }^{2}$ Oxford University Hospitals, Oxford, UK

${ }^{3}$ Cambridge University Hospitals NHS Foundation Trust, Cambridge, UK

${ }^{4}$ Northern Ireland Haemophilia Centre and Thrombosis Unit, Belfast City Hospital, Belfast, UK

${ }^{5}$ Department of Haematology, Barts and The London NHS Trust, London, UK

${ }^{6}$ Department of Haematology, City Hospitals Sunderland NHS Foundation Trust, Sunderland, UK

${ }^{7}$ King's Thrombosis Centre, King's College Hospital, London, UK

${ }^{8}$ Haemostasis \& Thrombosis Unit, Leicester Haemophilia Centre, The Leicester Royal Infirmary, Leicester, UK
}

${ }^{9}$ Department of Haematology, Warwick Hospital, Warwick, UK

${ }^{10}$ Department of Haematology, Glasgow Royal Infirmary, Glasgow, UK

Contributors RA and RR drafted the manuscript; DK revised the manuscript; TB, $\mathrm{GB}, \mathrm{LG}, \mathrm{SM}, \mathrm{RP}, \mathrm{SP}, \mathrm{PR}, \mathrm{CT}$ contributed to the manuscript. All authors approved the final manuscript. $R A$ is the guarantor.

Competing interests $R A, T B, G B, R P, S P, P R$ and $C T$ have received honoraria for lectures and/or consultancy work from Boehringer Ingelheim (BI). DK was an investigator on the RE-COVER Study sponsored by BI. The cost of Open Access was funded by an unrestricted educational grant from $\mathrm{BI}$ who had no editorial input into the paper.

Provenance and peer review Not commissioned; externally peer reviewed.

Open Access This is an Open Access article distributed in accordance with the Creative Commons Attribution Non Commercial (CC BY-NC 3.0) license, which permits others to distribute, remix, adapt, build upon this work non-commercially, and license their derivative works on different terms, provided the original work is properly cited and the use is non-commercial. See: http://creativecommons.org/ licenses/by-nc/3.0/

\section{REFERENCES}

1 Stangier J, Rathgen K, Stahle H, et al. The pharmacokinetics, pharmacodynamics and tolerability of dabigatran etexilate, a new oral direct thrombin inhibitor, in healthy male subjects. Br J Pharmacol 2007;64:292-303.

2 Connolly S, Ezekowitz M, Yusuf S, et al. the RE-LY Steering Committee and Investigators. Dabigatran versus warfarin in patients with atrial fibrillation. $N$ Engl J Med 2009;361:1139-51

3 Schulman S, Kearon C, Kakkar A, et al. for the RE-COVER Study Group. Dabigatran versus warfarin in the treatment of acute venous thromboembolism. $N$ Engl J Med 2009;361:2342-52.

4 Watanabe M, Siddiqui F, Qureshi Al. Incidence and management of ischaemic stroke and hemorrhage in patients on dabigatran etexilate treatment. Neurocrit Care 2012;16:203-9.

5 Cotton B, McCarthy J, Holcomb J. Acutely injured patients on dabigatran. N Eng J Med 2011;365:2039-40.

6 Harper P, Young L, Merriman E. Bleeding risk with dabigatran in the frail elderly. $N$ Eng J Med 2012;366:864-6.

7 NICE. Dabigatran etexilate for the prevention of stroke and systemic embolism in atrial fibrillation. 2012; http://guidance.nice.org.uk/TA/Wave21/10 (accessed 18 Sep 2012).

8 SMC. Dabigatran for the prevention of stroke and systemic embolisation in adult patients with non-valvular atrial fibrillation. 2011; http://www.scottishmedicines.org. uk/files/advice/dabigatran_Pradaxa_FINAL_August_2011_Amended_05.09. 11_for_website.pdf (accessed 18 Sep 2012).

9 Ageno W, Gallus A, Wittkowsky A, et al. Oral anticoagulant therapy: antithrombotic therapy and prevention of thrombosis, 9th ed: American College of Chest Physicians evidence-based clinical practice guidelines. Chest 2012;141:e44S-88S

10 Keeling D, Baglin T, Tait $C$, et al. Guidelines on oral anticoagulation with warfarin — fourth edition. Br J Haem 2011;154:311-24.

11 Lindahl T, Baghaei F, Fagerberg I, et al. Effects of the oral direct thrombin inhibitor dabigatran on five common coagulation assays. Thromb Haemost 2011;105:371-8.

12 Baglin T, Barrowcliffe T, Cohen A, et al. Guidelines on the use and monitoring of heparin. Br J Haem 2006;133:19-34.

13 Boehringer Ingelheim. Data on file.

14 Huisman M, Lip G, Diener HC, et al. Dabigatran etexilate for stroke prevention in patients with atrial fibrillation: resolving uncertainties in routine practice. Thromb Haemost 2012;107:838-47.

15 eMC. Summary of Product Characteristics: Pradaxa. 2012 (Last updated on eMC: 30/04/2012); http://www.medicines.org.uk/emc/medicine/24839/SPC/ (accessed 14 Sep 2012).

16 Stangier J, Feuring M. Using the HEMOCLOT direct thrombin inhibitor assay to determine plasma concentrations of dabigatran. Blood Coagul Fibrinolysis 2012;23:138-43.

17 van Ryn J, Litzenburger T, Waterman A, et al. Dabigatran anticoagulant activity is neutralized by an antibody selective to dabigatran in in vitro and in vivo models. J Am Col Cardiol 2011;57:E1130.

18 van Ryn J, Stangier J, Haertter S, et al. Dabigatran etexilate- a novel, reversible, oral direct thrombin inhibitor: interpretation of coagulation assays and reversal of anticoagulant activity. Thromb Haemost 2010;103:1116-27.

19 Pharmac N, Z Government. Guidelines for testing and perioperative management of dabigatran—for possible inclusion into local management protocols. 2011; http:// www.pharmac.govt.nz/2011/06/13/Dabigatran\%20testing\%20and\%20perioperative \%20management.pdf (accessed 18 Sep 2012).

20 Cushman M, Lim W, Zakai N. Clinical practice guide on anticoaguant dosing and management of anticoagulant-associated bleeding complications in adults. Am Soc Hemato/ 2011; http://www.hematology.org/Practice/Guidelines/2934.aspx (accessed 18 Sep 2012). 
21 van Ryn J, Sieger $\mathrm{P}$, Kink-Eiband $\mathrm{M}$, et al. Adsorption of dabigatran etexilate in water or dabigatran in pooled human plasma by activated charcoal in vitro. Blood 2009;114:Abstract 1065.

22 van Ryn J, Neubauer M, Flieg R, et al. Successful removal of dabigatran in flowing blood with an activated charcoal hemoperfusion column in an in vitro test system. Haematologica 2010;95(Suppl 2):Abstract 0700.

23 Henry D, Carless P, Moxey A, et al. Anti-fibrinolytic use for minimising perioperative allogeneic blood transfusion. Cochrane Database Syst Rev 2007;4:CD001886.

24 CRASH-2. Effects of tranexamic acid on death, vascular occlusive events, and blood transfusion in trauma patients with significant haemorrhage (CRASH-2): a randomised, placebo-controlled trial. Lancet 2010;376:23-32.

25 Blech S, Ebner T, Ludwig-Schwellinger E. The metabolism and disposition of the oral direct thrombin inhibitor, dabigatran, in humans. Drug Metab Dispos 2008;36:386-99.

26 Stangier J, Rathgen $\mathrm{K}$, Stahle $\mathrm{H}$, et al. Influence of renal impairment on the pharmacokinetic and pharmacodynamics of oral dabigatran etexilate. Clin Pharmacokin 2010;49:259-68.

27 Wagner FPH, Formella S, Wiegart E, et al. Effective elimination of dabigatran with haemodialysis: a phase I single centre study in patients with end stgae renal disease. Circulation 2011;124:Abstract 13303.

28 Pragst I, Zeitler S, Doerr B, et al. Reversal of dabigatran anticoagulation by prothrombin complex concentrate (Beriplex $\mathrm{P} / \mathrm{N}$ ) in a rabbit model. J Thromb Haemost 2012;10:1841-8.
29 van Ryn J, Schurer J, Kink-Eiband M, et al. The successful reversal of dabiagtran-induced bleeding by coagulation factor concentrates in a rat tail bleeding model do not correlate with ex vivo markers of anticoagulation. Blood 2011;118:Abstract 2316.

30 Zhou WSS, Illanes S, Liesz A, et al. Hemostatic therapy in experimental intracerbral hemorrhage associated with the direct thrombin inhibitor dabigatran. Stroke 2011:42:3594-9.

31 Eerenberg ES, Kamphuisen PW, Sijpkens MK, et al. Reversal of Rivaroxaban and Dabigatran by Prothrombin Complex Concentrate. Circulation 2011;124:1573-9.

32 Pragst I, Doerr B, Kaspereit $\mathrm{F}$, et al. Beriplex P/N reverses bleeeding in an acute renal injury model after dabiagtran overdose in rabbits. Pathophys Haemost Thromb 2010;37.

33 Turecek PL, Váradi K, Gritsch H, et al. FEIBA: mode of action. Haemophilia 2004;10:3-9.

34 Dager W. Reversing dabigatran with FEIBA in a patient with a transeptal perforation during cardiac ablation. Crit Care Med 2011;39:Abstract 867.

35 Hedner U, Lee CA. First 20 years with recombinant FVIla (NovoSeven). Haemophilia 2011;17:e172-82.

36 Levi M, Levy JH, Andersen HF, et al. Safety of recombinant activated factor VII in randomized clinical trials. N Engl J Med 2010;363:1791-800.

37 Gogarten W, Vandermeulen E, Van Aken H, et al. Regional anaesthesia and antithrombotic agents: recommendations of the European Society of Anaesthesiology. Eur J Anaesthesiol 2010;27:999-1015. 